International Mathematical Forum, 2, 2007, no. 45, 2237 - 2244

\title{
Solution of the Wave Equation by Homotopy Perturbation Method
}

\author{
J. Biazar ${ }^{a, 1}$ and H. Ghazvini ${ }^{a, b}$ \\ ${ }^{a}$ Department of Mathematics, Faculty of Sciences \\ P.O. Box 1914 P.C. 41938, Rasht, Iran \\ ${ }^{b}$ Department of Mathematics, School of Mathematical Sciences \\ Shahrood University of Technology \\ P.O. Box 316, P.C. 3619995161, Shahrood, Iran
}

\begin{abstract}
Homotopy perturbation method has been applied to solve many functional equations so far. In this article, we have uses this method to solve the wave equation, which governs numerous engineering experimentations, particularly in geosciences applications. Comparison of the results with those of Adomian's decomposition method [5] leads to significant consequences. Some special cases of the equation are solved as examples, to illustrate the reliable ness of the method.
\end{abstract}

Keywords: Homotopy perturbation method, Wave equation

\section{Introduction}

Since the governing equations on many experiments in engineering as well as science leads to the wave equation, this equation has attracted much attention, and solving the equation has been one of the interesting tasks for mathematicians. Analytical methods commonly used for solving the wave equation are very restricted and can be used in very special cases so they can not be used to solve equations of numerous realistic scenarios. Numerical techniques, which are commonly used, encounter difficulties in terms of the size of computational works needed and usually the round-off error causes the loss of accuracy. Homotopy perturbation method well addressed by He in [1-4] has been applied to

\footnotetext{
${ }^{1}$ Corresponding author:biazar@guilan.ac.ir(J. Biazar), hghazvini@guilan.ac.ir(H. Ghazvini).
} 
solve many functional equations. This method has proven to be very effective and results in considerable saving in computation time.

\section{Basic idea of homotopy perturbation method by $\mathrm{He}$}

To illustrate the basic ideas of the method, we consider nonlinear differential equation

$$
A(u)=f(r), \quad r \in \Omega
$$

with boundary conditions

$$
B\left(u, \frac{\partial u}{\partial n}\right)=0, \quad r \in \Gamma,
$$

where $A$ is a general differential operator, $B$ is a boundary operator, $f(r)$ is a known analytic function, and $\Gamma$ is the boundary of the domain $\Omega$. The operator $A$ can, generally speaking, be divided into two parts $L$ and $N$, where $L$ is linear, and $N$ is nonlinear operator. Eq. (1), therefore, can be rewritten as follows:

$$
L(u)+N(u)-f(r)=0 .
$$

By the homotopy technique [1], we construct a homotopy $v(r, p): \Omega \times[0,1] \rightarrow \mathbb{R}$ which satisfies

$$
H(v, p)=(1-p)\left[L(v)-L\left(u_{0}\right)\right]+p[A(v)-f(r)]=0, \quad p \in[0,1], \quad r \in \Omega
$$

or

$$
H(v, p)=L(v)-L\left(u_{0}\right)+p L\left(u_{0}\right)+p[N(v)-f(r)]=0,
$$

where $p \in[0,1]$ is an embedding parameter, $u_{0}$ is an initial approximation of Eq. (1), which satisfies the boundary conditions. Obviously, from Eq. (2) we have

$$
\begin{aligned}
& H(v, 0)=L(v)-L\left(u_{0}\right)=0 \\
& H(v, 1)=A(v)-f(r)=0
\end{aligned}
$$

The changing process of $p$ from zero to unity is just that of $v(r, p)$ from $u_{0}(r)$ to $u(r)$. In topology, this is called deformation and quantities $L(v)-L\left(u_{0}\right)$ and $A(v)-f(r)$ are called homotopic. Suppose, the solution of Eq. (2) can be written as a power series in $p$,

$$
v=v_{0}+p v_{1}+p^{2} v_{2}+\cdots .
$$

Setting $\mathrm{p}=1$ results in approximate solution of Eq. (1)

$$
u=\lim _{p \rightarrow 1} v=v_{0}+v_{1}+v_{2}+\ldots
$$




\section{The homotopy perturbation to wave equa- tions}

Consider the following general form of wave equation with the indicated initial and boundary conditions:

$$
\frac{\partial^{2} u}{\partial t^{2}}=A(x, y, z, t) \frac{\partial^{2} u}{\partial x^{2}}+B(x, y, z, t) \frac{\partial^{2} u}{\partial y^{2}}+C(x, y, z, t) \frac{\partial^{2} u}{\partial z^{2}}+D(x, y, z, t)
$$

With the initial conditions

$$
u(x, y, z, 0)=f(x, y, z), \frac{\partial u}{\partial t}(x, y, z, 0)=g(x, y, z)
$$

Or the following boundary conditions

$$
u(L, y, z, t)=f_{1}(y, z, t), \frac{\partial u}{\partial x}(L, y, z, t)=g_{1}(x, y, z)
$$

For solving Eq. (3) with initial conditions (4) according to the homotopy perturbation, we construct the following homotopy:

$$
(1-p)\left(\frac{\partial^{2} v}{\partial t^{2}}-\frac{\partial^{2} u_{0}}{\partial t^{2}}\right)+p\left(\frac{\partial^{2} v}{\partial t^{2}}-A \frac{\partial^{2} v}{\partial x^{2}}-B \frac{\partial^{2} v}{\partial y^{2}}-C \frac{\partial^{2} v}{\partial z^{2}}-D\right)=0
$$

or

$$
\frac{\partial^{2} v}{\partial t^{2}}-\frac{\partial^{2} u_{0}}{\partial t^{2}}=p\left(A \frac{\partial^{2} v}{\partial x^{2}}+B \frac{\partial^{2} v}{\partial y^{2}}+C \frac{\partial^{2} v}{\partial z^{2}}+D-\frac{\partial^{2} u_{0}}{\partial t^{2}}\right)
$$

with initial approximation $u_{0}=f(x, y, z)+g(x, y, z) t$. Suppose the solution of Eq. (6) has the form

$$
v=v_{0}+p v_{1}+p^{2} v_{2}+\cdots
$$

Substituting (7) into (6), and equating the terms with the identical powers of $p$,

$$
\begin{aligned}
& p^{0}: \frac{\partial^{2} v_{0}}{\partial t^{2}}-\frac{\partial^{2} u_{0}}{\partial t^{2}}=0 \\
& p^{1}: \frac{\partial^{2} v_{1}}{\partial t^{2}}=A \frac{\partial^{2} v_{0}}{\partial x^{2}}+B \frac{\partial^{2} v_{0}}{\partial y^{2}}+C \frac{\partial^{2} v_{0}}{\partial z^{2}}+D-\frac{\partial^{2} u_{0}}{\partial t^{2}}, \quad v_{1}(x, y, z, 0)=\frac{\partial v_{1}}{\partial t}(x, y, z, 0) \\
& p^{2}: \frac{\partial^{2} v_{2}}{\partial t^{2}}=A \frac{\partial^{2} v_{1}}{\partial x^{2}}+B \frac{\partial^{2} v_{1}}{\partial y^{2}}+C \frac{\partial^{2} v_{1}}{\partial z^{2}}, \quad v_{2}(x, y, z, 0)=\frac{\partial v_{2}}{\partial t}(x, y, z, 0)
\end{aligned}
$$


For simplicity we always take $v_{0}=u_{0}=f(x, y, z)+g(x, y, z) t$. So we have

$$
\begin{aligned}
& v_{1}=\int_{0}^{t} \int_{0}^{t}\left(A(x, y, z, \xi) \frac{\partial^{2} v_{0}}{\partial x^{2}}+B(x, y, z, \xi) \frac{\partial^{2} v_{0}}{\partial y^{2}}+C(x, y, z, \xi) \frac{\partial^{2} v_{0}}{\partial z^{2}}+D(x, y, z, \xi)\right) d \xi d t \\
& v_{2}=\int_{0}^{t} \int_{0}^{t}\left(A(x, y, z, \xi) \frac{\partial^{2} v_{1}}{\partial x^{2}}+B(x, y, z, \xi) \frac{\partial^{2} v_{1}}{\partial y^{2}}+C(x, y, z, \xi) \frac{\partial^{2} v_{1}}{\partial z^{2}}\right) d \xi d t
\end{aligned}
$$

The approximate solution of (3), therefore, can be obtained by setting $p=1$

$$
u=v_{0}+v_{1}+v_{2}+\cdots .
$$

Similarly, to solve Eq. (3) with boundary condition (5) we construct the following homotopy

$$
\frac{\partial^{2} v}{\partial x^{2}}-\frac{\partial^{2} u_{0}}{\partial x^{2}}=p\left(\frac{1}{A} \frac{\partial^{2} v}{\partial t^{2}}-\frac{B}{A} \frac{\partial^{2} v}{\partial y^{2}}-\frac{C}{A} \frac{\partial^{2} v}{\partial z^{2}}-\frac{D}{A}-\frac{\partial^{2} u_{0}}{\partial x^{2}}\right)
$$

with the initial approximation $v_{0}=u_{0}=f_{1}(y, z, t)+g_{1}(y, z, t)(x-L)$. Suppose the solution of Eq. (10) has the form (7), according to scheme mentioned above we have

$$
\begin{aligned}
& p^{0}: \frac{\partial^{2} v_{0}}{\partial x^{2}}-\frac{\partial^{2} u_{0}}{\partial x^{2}}=0 \\
& p^{1}: \frac{\partial^{2} v_{1}}{\partial x^{2}}=\frac{1}{A} \frac{\partial^{2} v_{0}}{\partial t^{2}}-\frac{B}{A} \frac{\partial^{2} v_{0}}{\partial y^{2}}-\frac{C}{A} \frac{\partial^{2} v_{0}}{\partial z^{2}}-\frac{D}{A}-\frac{\partial^{2} u_{0}}{\partial x^{2}}, v_{1}(L, y, z, t)=\frac{\partial v_{1}}{\partial x}(L, y, z, t)=0 \\
& p^{2}: \frac{\partial^{2} v_{2}}{\partial x^{2}}=\frac{1}{A} \frac{\partial^{2} v_{1}}{\partial t^{2}}-\frac{B}{A} \frac{\partial^{2} v_{1}}{\partial y^{2}}-\frac{C}{A} \frac{\partial^{2} v_{1}}{\partial z^{2}}, v_{2}(L, y, z, t)=\frac{\partial v_{2}}{\partial x}(L, y, z, t)=0 \\
& \vdots
\end{aligned}
$$

Accordingly we have:

$$
\begin{aligned}
& v_{1}=\int_{L}^{x} \int_{L}^{x}\left(\frac{1}{A(\xi, y, z, t)} \frac{\partial^{2} v_{0}}{\partial t^{2}}-\frac{B(\xi, y, z, t)}{A(\xi, y, z, t)} \frac{\partial^{2} v_{0}}{\partial y^{2}}-\frac{C(\xi, y, z, t)}{A(\xi, y, z, t)} \frac{\partial^{2} v_{0}}{\partial z^{2}}-\frac{D(\xi, y, z, t)}{A(\xi, y, z, t)}\right) d \xi d x \\
& v_{2}=\int_{L}^{x} \int_{L}^{x}\left(\frac{1}{A(\xi, y, z, t)} \frac{\partial^{2} v_{1}}{\partial t^{2}}-\frac{B(\xi, y, z, t)}{A(\xi, y, z, t)} \frac{\partial^{2} v_{1}}{\partial y^{2}}-\frac{C(\xi, y, z, t)}{A(\xi, y, z, t)} \frac{\partial^{2} v_{1}}{\partial z^{2}}\right) d \xi d x
\end{aligned}
$$

Setting $p=1$, results in approximation solution of E.q (3),

$$
u=\lim _{p \rightarrow 1} v=v_{0}+v_{1}+v_{2}+\cdots
$$

\section{Examples}

To illustrate the method and to show the ability of the method so examples are presented here. 
Example 1. Consider the wave equation with the following initial conditions [5]:

$$
\left\{\begin{array}{l}
\frac{\partial^{2} u}{\partial t^{2}}=A \frac{\partial^{2} u}{\partial x^{2}}+B \frac{\partial^{2} u}{\partial y^{2}}+C \frac{\partial^{2} u}{\partial z^{2}}+D \\
u(x, y, z, 0)=a_{1} x+a_{2} x^{2}+b_{1} y+b_{2} y^{2}+c_{1} z+c_{2} z^{2} \\
\frac{\partial u}{\partial t}(x, y, z, 0)=a_{1}^{\prime} x+a_{2}^{\prime} x^{2}+b_{1}^{\prime} y+b_{2}^{\prime} y^{2}+c_{1}^{\prime} z+c_{2}^{\prime} z^{2}
\end{array}\right.
$$

Where A, B, C and D are constant. Suppose $u=u_{0}+p u_{1}+p^{2} u_{2}+\cdots$, according to (6), we have

$$
\frac{\partial^{2} u}{\partial t^{2}}-\frac{\partial^{2} u_{0}}{\partial t^{2}}=p\left(A \frac{\partial^{2} u}{\partial x^{2}}+B \frac{\partial^{2} u}{\partial y^{2}}+C \frac{\partial^{2} u}{\partial y^{2}}+D-\frac{\partial^{2} u_{0}}{\partial t^{2}}\right)
$$

Beginning with

$$
u_{0}=a_{1} x+a_{2} x^{2}+b_{1} y+b_{2} y^{2}+c_{1} z+c_{2} z^{2}+\left(a_{1}^{\prime} x+a_{2}^{\prime} x^{2}+b_{1}^{\prime} y+b_{2}^{\prime} y^{2}+c_{1}^{\prime} z+c_{2}^{\prime} z^{2}\right) t
$$

From (8) we have

$$
\begin{gathered}
\frac{\partial^{2} u_{1}}{\partial t^{2}}=2\left(A a_{2}+B b_{2}+C c_{2}\right)+2\left(A a_{2}^{\prime}+B b_{2}^{\prime}+C c_{2}^{\prime}\right) t+D, \\
u_{1}(x, y, z, 0)=\frac{\partial u_{1}}{\partial t}(x, y, z, 0)=0
\end{gathered}
$$

And we derive the following results,

$$
u_{1}(x, y, z, t)=\left(A a_{2}+B b_{2}+C c_{2}+\frac{1}{2} D\right) t^{2}+\left(A a_{2}^{\prime}+B b_{2}^{\prime}+C c_{2}^{\prime}\right) \frac{t^{3}}{3}
$$

So the first-order approximate solution is

$$
\begin{aligned}
u= & u_{0}+u_{1}=a_{1} x+a_{2} x^{2}+b_{2} y^{2}+c_{1} z+c_{2} z^{2}+\left(a_{1}^{\prime} x+a_{2}^{\prime} x^{2}+b_{1}^{\prime} y+b_{2}^{\prime} y^{2}+c_{1}^{\prime} z+c_{2}^{\prime} z^{2}\right) t \\
& +\left(A a_{2}+B b_{2}+C c_{2}+\frac{1}{2} D\right) t^{2}+\left(A a_{2}^{\prime}+B b_{2}^{\prime}+C c_{2}^{\prime}\right) \frac{t^{3}}{3}
\end{aligned}
$$

which is an exact solution.

Example 2. Let us solve the following partial differential equation [5]:

$$
\left\{\begin{array}{l}
\frac{\partial^{2} u}{\partial t^{2}}=\frac{1}{6}\left(x^{2} \frac{\partial^{2} u}{\partial x^{2}}+y^{2} \frac{\partial^{2} u}{\partial y^{2}}+z^{2} \frac{\partial^{2} u}{\partial z^{2}}\right) \\
u(x, y, z, 0)=x^{2} y^{2} z^{2} \\
\frac{\partial u}{\partial t}(x, y, z, 0)=-x^{2} y^{2} z^{2}
\end{array}\right.
$$

Its homotopy can be written down as follows

$$
\frac{\partial^{2} u}{\partial t^{2}}-\frac{\partial^{2} u_{0}}{\partial t^{2}}=p\left(\frac{1}{6}\left(x^{2} \frac{\partial^{2} u}{\partial x^{2}}+y^{2} \frac{\partial^{2} u}{\partial y^{2}}+z^{2} \frac{\partial^{2} u}{\partial z^{2}}\right)-\frac{\partial^{2} u_{0}}{\partial t^{2}}\right)
$$


Beginning with $u_{0}=x^{2} y^{2} z^{2}-x^{2} y^{2} z^{2} t$, from (6), we have

$$
\begin{aligned}
& \frac{\partial^{2} u_{1}}{\partial t^{2}}=x^{2} y^{2} z^{2}-x^{2} y^{2} z^{2} t, \quad u_{1}(x, y, z, 0)=\frac{\partial u_{1}}{\partial t}(x, y, z, 0)=0 \\
& \frac{\partial^{2} u_{2}}{\partial t^{2}}=x^{2} y^{2} z^{2} \frac{t^{2}}{2 !}-x^{2} y^{2} z^{2} \frac{t^{3}}{3 !}, \quad u_{2}(x, y, z, 0)=\frac{\partial u_{2}}{\partial t}(x, y, z, 0)=0 \\
& \vdots \\
& \frac{\partial^{2} u_{n}}{\partial t^{2}}=x^{2} y^{2} z^{2} \frac{t^{2 n-2}}{(2 n-2) !}-x^{2} y^{2} z^{2} \frac{t^{2 n-1}}{(2 n-1) !}, \quad u_{n}(x, y, z, 0)=\frac{\partial u_{n}}{\partial t}(x, y, z, 0)=0
\end{aligned}
$$

Applying the inverse of differentiation operator, we have

$$
\begin{aligned}
& u_{1}(x, y, z, t)=x^{2} y^{2} z^{2}\left(\frac{t^{2}}{2 !}-\frac{t^{3}}{3 !}\right) \\
& u_{2}(x, y, z, t)=x^{2} y^{2} z^{2}\left(\frac{t^{4}}{4 !}-\frac{t^{5}}{5 !}\right) \\
& \vdots \\
& u_{n}(x, y, z, t)=x^{2} y^{2} z^{2}\left(\frac{t^{2 n}}{(2 n) !}-\frac{t^{2 n+1}}{(2 n+1) !}\right)
\end{aligned}
$$

Thus we have $u(x, y, z, t)=x^{2} y^{2} z^{2} \sum_{n=0}^{\infty}(-1)^{n} \frac{t^{n}}{n !}=x^{2} y^{2} z^{2} e^{-t}$, which is an exact solution.

Example 3. Consider the following partial equation with specified initial conditions [5] :

$$
\left\{\begin{array}{l}
\frac{\partial^{2} u}{\partial t^{2}}=e^{-x t} \frac{\partial^{2} u}{\partial x^{2}}+e^{-y t} \frac{\partial^{2} u}{\partial y^{2}}+e^{-z t} \frac{\partial^{2} u}{\partial z^{2}} \\
u(x, y, z, 0)=x^{2}+y^{2}+z^{2} \\
\frac{\partial u}{\partial t}(x, y, z, 0)=0
\end{array}\right.
$$

Homotomy perturbation method consist of the following scheme:

$$
\frac{\partial^{2} u}{\partial t^{2}}-\frac{\partial^{2} u_{0}}{\partial t^{2}}=p\left(e^{-x t} \frac{\partial^{2} u}{\partial y^{2}}+e^{-y t} \frac{\partial^{2} u}{\partial y^{2}}+e^{-2 t} \frac{\partial^{2} u}{\partial z^{2}}-\frac{\partial^{2} u_{0}}{\partial t^{2}}\right)
$$

Beginning with $u_{0}(x, y, z, t)=x^{2}+y^{2}+z^{2}$, by using (8) and (9) we have

$$
\begin{gathered}
u_{1}(x, y, z, t)= \\
\frac{2\left(\left(e^{-x t}(1+x)-1\right) y^{2} z^{2}+\left(e^{-y t}(1+y)-1\right) x^{2} z^{2}+\left(e^{-z t}(1+z)-1\right) y^{2} x^{2}\right)}{x^{2} y^{2} z^{2}} .
\end{gathered}
$$

And $u_{2}$ can be calculated similarly. Considering $u \approx u_{0}+u_{1}+u_{2}$, therefore,

$$
\begin{aligned}
& u(x, y, z, t) \approx \frac{1}{y^{6} x^{6} z^{6}}\left(-2 t z^{6} y^{6} x^{5}-24 e^{(-y t)} z^{6} x^{6} y+2 z^{5} y^{6} x^{6}+2 e^{(-2 x t)} t^{2} y^{6} x^{2} z^{6}+24 e^{(-2 z t)} y^{6} x^{6} z\right. \\
& +24 e^{(-2 x t)} y^{6} x z^{6}+24 t e^{(-z t)} y^{6} x^{6} z-40 e^{(-z t)} y^{6} x^{6}-2 t z^{5} y^{6} x^{6}+40 e^{(-2 y t)} z^{6} x^{6} \\
& +40 e^{(-2 x t)} z^{6} y^{6}+12 t e^{(-2 z t)} y^{6} x^{6} z^{2}+12 t e^{(-2 x t)} y^{6} z^{6} x^{2}+16 t e^{(-2 x t)} y^{6} z^{6} x \\
& +16 t e^{(-2 z t)} y^{6} x^{6} z+12 t e^{(-2 y t)} y^{2} z^{6} x^{6}+y^{8} z^{6} x^{6}+z^{8} y^{6} x^{6}+x^{8} z^{6} y^{6} \\
& +16 t e^{(-2 y t)} z^{6} x^{6} y+24 e^{(-2 y t)} z^{6} x^{6} y-6 t y^{6} z^{6} x^{6}+2 y^{5} z^{6} x^{6}+y^{6} z^{6} x^{6}+12 t e^{(-z t)} z^{2} x^{6} y^{2} \\
& -2 t y^{5} z^{6} x^{6}+24 t e^{(-x t)} z^{6} y^{6} x+40 e^{(-2 z t)} x^{6} y^{6}+12 t e^{(-x t)} z^{6} y^{6} x^{2}+2 e^{(-2 y t)} t^{2} z^{6} y^{2} x^{6} \\
& +2 e^{(-2 y t)} t^{2} z^{6} y^{3} x^{6}-40 e^{(-x t)} z^{6} y^{6}+2 z^{6} y^{6} x^{5}-40 e^{(-y t)} z^{6} x^{6}-24 e^{(-x t)} z^{6} y^{6} x \\
& -24 e^{(-z t)} x^{6} y^{6} z+12 t e^{(-y t)} z^{6} y^{2} x^{6}+24 t e^{(-y t)} z^{6} y x^{6}-6 t^{2} e^{(-z t)} z^{2} y^{6} x^{6} \\
& -6 t^{2} e^{(-x t)} z^{6} y^{6} x^{2}-6 t^{2} e^{(-y t)} z^{3} y^{6} x^{6}+6 t^{2} e^{(-y t)} z^{6} y^{6} x^{6}+t^{2} e^{(-z t)} z^{6} y^{6} x^{6} \\
& +t^{2} e^{(-y t)} z^{6} y^{6} x^{6}+2 t^{2} e^{(-2 z t)} z^{3} y^{6} x^{6}+2 t^{2} e^{(-2 z t)} z^{2} y^{6} x^{6}+2 t^{2} e^{(-2 x t)} z^{6} y^{6} x^{3}
\end{aligned}
$$


Example 4. Consider the following partial differential equation with the following boundary conditions:

$$
\left\{\begin{array}{l}
\frac{\partial^{2} u}{\partial t^{2}}=A \frac{\partial^{2} u}{\partial x^{2}}+B \frac{\partial^{2} u}{\partial y^{2}}+C \frac{\partial^{2} u}{\partial z^{2}}+D \\
u(0, y, z, t)=a_{1} y+a_{2} y^{2}+b_{1} z+b_{2} z^{2} \\
\frac{\partial u}{\partial x}(0, y, z, t)=\left(a_{1}^{\prime} y+a_{2}^{\prime} y^{2}+b_{1}^{\prime} z+b_{2}^{\prime} z^{2}\right) t
\end{array}\right.
$$

Where A, B, C and D are constant. From (10) we derive

$$
\frac{\partial^{2} u}{\partial x^{2}}-\frac{\partial^{2} u_{0}}{\partial x^{2}}=p\left(\frac{1}{A} \frac{\partial^{2} u}{\partial t^{2}}+\frac{B}{A} \frac{\partial^{2} u}{\partial y^{2}}-\frac{C}{A} \frac{\partial^{2} u}{\partial z^{2}}-\frac{D}{A}-\frac{\partial^{2} u_{0}}{\partial x^{2}}\right)
$$

By imposing $u_{0}(x, y, z, t)=a_{1} y+a_{2} y^{2}+b_{1} z+b_{2} z^{2}+\left(a_{1}^{\prime} y+a_{2}^{\prime} y^{2}+b_{1}^{\prime} z+b_{2}^{\prime} z^{2}\right) x t$ From (11), we have

$$
\begin{gathered}
\frac{\partial^{2} u_{1}}{\partial x^{2}}=-\frac{1}{A}\left(2\left(a_{2} B+b_{2} C\right)+D-2\left(a_{2}^{\prime} B+b_{2}^{\prime} C\right) x t\right) \\
u_{1}(x, y, z, t)=\frac{\partial u_{1}}{\partial x}(0, y, z, t)=0
\end{gathered}
$$

The solution reads

$$
u_{1}(x, y, z, t)=-\frac{1}{A}\left(\left(a_{2} B+b_{2} C+\frac{1}{2} D\right) x^{2}+\left(a_{2}^{\prime} B+b_{2}^{\prime} C\right) \frac{t x^{3}}{3}\right) .
$$

It can be easily shown $u_{i}=0$, for $i=2,3, \ldots$ and so an exact solution as follows

$$
\begin{aligned}
& u(x, y, z, t)=u_{0}+u_{1}=a_{1} y+a_{2} y^{2}+b_{1} z+b_{2} z^{2}+\left(a_{1}^{\prime} y+a_{2}^{\prime} y^{2}+b_{1}^{\prime} z+b_{2}^{\prime} z^{2}\right) x t \\
& \left.-\frac{1}{A}\left(\left(a_{2} B+b_{2} C+\frac{1}{2} D\right) x^{2}+a_{2}^{\prime} B+b_{2}^{\prime} C\right) \frac{t x^{3}}{3}\right) .
\end{aligned}
$$

Example 5. Let us solve the following partial differential equation:

$$
\left\{\begin{array}{l}
\frac{\partial^{2} u}{\partial t^{2}}=x \frac{\partial^{2} u}{\partial x^{2}}+y \frac{\partial^{2} u}{\partial y^{2}}+z \frac{\partial^{2} u}{\partial z^{2}} \\
u(1, y, z, t)=y z t^{2} \\
\frac{\partial u}{\partial t}(1, y, z, t)=-y z t^{2}
\end{array}\right.
$$

Its homotopy can be written down as follows:

$$
\frac{\partial^{2} u}{\partial x^{2}}-\frac{\partial^{2} u_{0}}{\partial x^{2}}=p\left(\frac{1}{x} \frac{\partial^{2} u}{\partial t^{2}}-\frac{y}{x} \frac{\partial^{2} u}{\partial y^{2}}-\frac{z}{x} \frac{\partial^{2} u}{\partial z^{2}}-\frac{\partial^{2} u_{0}}{\partial x^{2}}\right)
$$


Beginning with $u_{0}=2 y z t^{2}-x y z t^{2}$, and using (11), (12) and (13) we get $u_{1}(x, y, z, t)=4 x y z \operatorname{lm} x-2 x y z-x^{2} y z+3 y z, u_{i}(x, y, z, t)=0, i=2,3, \ldots$.

Thus we have

$$
u(x, y, z, t)=u_{0}+u_{1}=2 y z t^{2}-x y z t^{2}+4 x y z \ln x-x^{2} y z-2 x y z+3 y z,
$$

which is an exact solution. It's worth to point out that, the results in examples 1, 2 and 3 are exactly the same as Adomain decomposition method [5].

\section{Conclusions}

The main goal of this article, was derive an analytical approximation solution for the wave equation. We have achieved this goal by applying homotopy perturbation method. In some examples we have derive an exact solution but in others a very accurate analytical approximation. Compared with Adomian decomposition method [5], the present method there is no need to calculate Adomian polynomials. The small size of computations, in comparison with the computational size required in numerical methods and the rapid convergence shows that the homotopy perturbation method is reliable, and introduces a significant improvement in solving the wave equation over existing methods. Maple 10 is used to carry computations.

\section{References}

[1] J.H. He, Homotopy perturbation technique, Computer Methods in Applied Mechanics and Engineering 178 (1999) 257-262.

[2] J.H. He, A coupling method of homotopy technique and perturbation technique for nonlinear problems, International Journal of Non-Linear Mechanics 35 (1) (2000) 37-43.

[3] J.H. He, A modified perturbation technique depending upon an artificial parameter, Mechanical 35 (2000) 299-311.

[4] J.H. He, Homotopy perturbation method for solving boundary value problems, Physics Letters A 350 (2006) 87-88.

[5] J.Biazar, R. Islam, solution of wave equation by Adomian decomposition method and the restrictions of the method, Applied Mathematics and Computation 149 (2004) 807-814.

\section{Received: November 21, 2006}

